\title{
Digitalization of the power business: How to make this work?
}

\author{
A.B. Svendsen \& T. Tollefsen \\ Promaps Technology, Norway \\ T. Gjengedal \\ UiT-The Arctic University of Norway, Norway \\ M. Goodwin \\ UiA-The University of Agder, Norway \\ S. Antonsen \\ NTNU-The Norwegian University of Science and Technology, Norway
}

\begin{abstract}
As a result of the digitalization of the power business in Norway and Europa, a lot of new possibilities and challenges arise. In 2014 an expert committee one outlined a proposal for the future grid company structure in Norway (Reiten, 2014). In addition, new technologies are being implemented in the system. Wind power, solar power, un-regulated small hydro power production, battery storage domestic and industrial and electrification of transport. Transmission System Operators (TSOs) have a responsibility to supply industry and communities with reliable electric power. However, the operators have been virtually blind to slowly occurring changes in the load profile that reduce the expected regularity of the power supply. This paper will focus on the possibilities and challenges the power business are facing. The paper will describe what technologies is needed i.e Real time probabilistic risk calculations, artificial intelligence, machine learning and smart grid technology. The main question is: can the power business and the introduction of new system tools manage without probabilistic risk calculation for making use of the digitalization and the corresponding big data?
\end{abstract}

\section{INTRODUCTION}

\subsection{History of the electric grid}

Modern Norway was built and industrialized by the fact that we managed to utilize rivers and waterfalls for power generation. Hydropower is still the cornerstone of the Norwegian power system, but wind power and solar energy is becoming an increasing part of the energy system. The grid has been developed over 150 years since the first small hydro plants were installed to supply small local industries. Hydro power plants were constructed over time as the industrial development moved forward. Initially the generation supplied local and regional consumers, but as transmission technology developed regions were connected via high voltage lines.

Now, the main grid is the most important part of the grid system, as failure here could mean power outage for very many consumers. The main grid was built largely from the 1950s to the 1980s. However, regional islands existed until 1994 when the main grid was finally established throughout Norway. Deregulation and competition was introduced in 1991 with the purpose of improving the socio-economic efficiency in the energy sector Now, the Norwegian main grid is aging and in the process of being replaced and upgraded by construction of new $420 \mathrm{kV}$ lines in combination with digitalization of the power system. (Statnett, 2017).

\subsection{The change in the power system, smart grid, solar, wind, battery etc.}

The energy system is a critical part of a well-functioning society. Norway is largely electrified and power transmission is an important prerequisite for value creation.

Although the power grids are largely built as before, the power system changes at a rapid pace. Hence, the Transmission System Operator (TSO) must be an enabler and be prepared for the future. The Norwegian aging main grid is in the process of being replaced and upgraded. The power grid takes a long time to plan and build, and have a long lead time, which contrasts strongly with an energy sector in a rapid change. The load is increasing and more generation is being installed. The Green Certificate Scheme provides incentives to expand renewable power generation, including small scale 
hydropower, wind power and solar power. Implementation of Automatic Meter Reading and Control Systems at the consumer level will allow for activation of consumer flexibility. Consumption patterns in the energy sector are changing rapidly (NVE, 2016).

Hence, the growing expansion of renewable energy and activation of flexible loads increases the complexities in balancing generation and demand in the power system. The energy-shifting and fast-ramping capability of energy storage has led to increasing interests in batteries to facilitate the integration of renewable resources (Amrouche, 2016).

The future power system will become even more dynamic and the need for real time information for monitoring the system status and for taking the proper control actions are increasing

\subsection{The need for a solution}

Global challenges regarding energy and climate change, the environment, safety, technology and renewable solutions, use and conservation of energy, use of batteries and the connection of electrical vehicles requires greater effort. The changing landscape of the power and utilities industry is resulting in new expectations for IT. Transmission system operators are struggling to fulfil their traditional mission of maintaining security of supply in a rapidly evolving environment driven by digitalization. Digital transformation is something that has become a common trend, and it is one that has reached the Power \& Utilities sector moving rather quickly (Digitalization \& Energy, 2016). The physical power system cannot function effectively without a well-functioning power market with smart ICT systems. The power system must be able to cope with the increasing variability in load and generation. Hence, in a complex power system, new solutions in the field of ICT and new market models are required to ensure the reliability and security of supply, to ensure that the transmission capacity is optimally utilized and that control actions are taken when needed.

\section{DIGITALIZATION OF THE POWER BUSINESS - THE SOLUTION?}

\subsection{The goal with digitalization}

The concept of a Digital Power System (DPS) has been discussed for many years. The DPS may be defined like the digital power system being the digital, figuration and real-time description and reappearance of physical structure, technical characteristic, management system as well as personal information system of a real power system which is in operation. The DPS will be able to make a significant contri- bution to administrating and decision-making more scientifically (Chakrabortty, 2017).

The share unregulated renewable power generation is rising and the power system is changing rapidly. Changes like this must be able to handle tomorrow's energy system. Hence, the reasons and goals for implementing the digital power systems are multiple:

- Monitoring g and controlling all components by equipping them by sensors

- Measure the condition of the power system flows, angle differences, stability margins, and hence the reliability and security

- improving security and stability online, online making and implementing economical operation strategy and carrying out emergency and anti-fault control, etc.

- Better utilization of the facilities

- Precise state information results in increased capacity and fewer faults

- More efficient maintenance and increased lifetime

In the end, the primary goal is to increase the value creation while maintaining the reliability and security of the system.

\subsection{Big data}

Data has always been an important asset in every industry. Since the early days of the information age, business intelligence and descriptive statistics have been used as the standard tools for extracting information and make important decisions from all kinds of collected data. However, as the cost of collecting, storing, and processing data has been dropping exponentially, the amount and the diversity of the data has reached the point where traditional approaches are no longer feasible. The term Big Data is often used to refer to any data that requires new techniques and tools in order for it to be processed and analyzed. Big Data could also be looked from the point of view of the new set of technologies that are helping to solve the challenges in collecting, managing, and analyzing Big Data. These technologies include cloud computing and cluster computing for data storage and manipulation, Artificial Intelligence (AI) and machine learning for data analysis (L'Heureux, 2017).

As in many other sectors big data analytics and machine learning are also getting involved in the energy sector and tools are being developed. They are for example used to forecast electricity demand at substation level, segment customers based on their power consumption patterns, implement demand response strategies, for power system condition monitoring and controls.

The value of big data may come from several use cases: as a source of analytics, as a source for control actions and as an enabler for new products and serv- 
ices. An energy company could e.g. track, collect, and store all available data from their system from customers, from components, from system data, from GPS trails to geographical and meteorological data, then combine them together and use big data analytics to produce high value actionable insights and controls.

Use of big data technologies may also open up completely new business models and introduce new products and services in the energy sector.

\subsection{Smart grid}

The smart grid would be an enhancement of the electrical grid, using two-way communications and distributed intelligent devices (Smart Grids European Technology Platform, 2011). Two-way flows of electricity and information could improve the delivery network. A smart grid would allow the power industry to observe and control parts of the system at higher resolution in time and space. One of the purposes of the smart grid is real time information exchange to make operation as efficient as possible. It would allow management of the grid on all time scales from high-frequency switching devices on a microsecond scale, to wind and solar output variations on a minute scale, to the future effects of the carbon emissions generated by power production on a decade scale.

The management system in smart grid is the subsystem that provides advanced management and control services. Most of the focus aim to improve energy efficiency, demand profile, utility, flexibility, cost, based on the infrastructure by using optimization, machine learning and game theory. Within the advanced infrastructure framework of smart grid, more and more new management services and applications are expected to emerge and eventually revolutionize consumers' daily lives.

The protection system of a smart grid provides grid reliability analysis, failure protection, and security and privacy protection services. While the additional communication infrastructure of a smart grid provides additional protective and security mechanisms, it also presents a risk of external attack and internal failures (Pandey, 2017).

\section{POWER SYSTEM OPERATION}

\subsection{Balancing the system}

Electricity must be produced at the same time as the power is consumed. In addition, the production must be equal to the power consumed. This is called the instantaneous balance of the power system. The power market is the central tool for balancing supply and demand for power. The results of the daily pricing calculation in the dayahead market are the basis for the Norwegian TSO Statnett's planning and maintenance of current balance in the following operating day. The continuous balancing of production and consumption is very important for the reliability of the system. In case of imbalances, system administrators implement measures to restore the balance, such as adjusting output or consumption.

Statnett has been given the system responsibility in the Norwegian power system. System Requirements in the Power System (Regulations on system responsibility in the power system, 2002) emphasize that the system operator shall provide frequency regulation, ensure instantaneous balance in the power system, develop market solutions that contribute to the efficient development and utilization of the power system, and to the greatest extent possible use of instruments based on market principles. The System Responsible company coordinates the operation of the power system, provides for the determination of capacity for the market, bottleneck handling and trade with other countries.

A well-designed power system has the following characteristics:

- Provide all consumption regardless of geographical location

- Provide consumption at all times

- Must be able to handle variability in consumption and production

- Supply must be of good quality and meet defined quality requirements

- Must be based on economic 'optimal' principle

- Must meet required and defined security goals

The delivered power must meet certain minimum delivery quality requirements. The following determines the quality:

- System frequency must be kept around the specified $50 \mathrm{~Hz}$ with variation within $+-0,1 \mathrm{~Hz}$

- The voltages are kept within narrow, prescribed limits around the normal value. Generally, the voltage variation should be within $+-10 \%$ (or $5 \%$ in some systems)

To ensure that voltages and frequencies are kept within their limits, voltage and frequency regulation is required for efficient operation of the power system (Gjengedal, 2017).

\subsection{Traditionally operation and planning $\mathrm{N}-\mathrm{I}$}

Operating the network according to the $\mathrm{N}-1$ criterion means that failure of a component does not result in interruptions in the supply to the end user. It is referred to as reduced reliability when the N-1 criterion is no longer met, in cases where it should normally be met. Statnett as a system administrator has the means through the system liability regulation (Regulations on system responsibility in the power system, 2002) to be able to change the grids configurations, as well as demand up-or 
down regulation of production. Such means can help ensure operation according to the $\mathrm{N}-1$ criterion. However, the authorities are not requiring that the main network should meet the N-1 operation safety at all times.

\subsection{Power system operation challenges}

In power system operation, traditionally slowchanging and predictable parameters are now changing fast. In addition, new system parameters are being introduced in the power system. This will influence the inherent properties of the system as well as the risk for outages. While the power system complexity is increasing, the operational available response time is decreasing fast. Example: The introduction of solar and wind production represents a power production which is difficult to manage when the wind stops or clouds cover the sun.

Combine this with a high degree of automation and smart grid technology, and the existing operational «know how» may not be sufficient to deal with the properties of the current and future power system.

What the neighboring power system is doing, will affect the current power system in regards of dynamics and risk. Until now power system operators have evaluated the system risk for loss of load qualitative, based on experience. This "gut feeling" based on such an experience approach, will not be valid in the future without nurturing new skills and competencies.

The first step is to be able to assess the system risk level equally for each power company. The only way to achieve this is by assessing this quantitatively with probabilistic approach.

The power business has lacked tools to evaluate quantitatively the system risk level in near realtime, and to assess possible risk reducing actions. This challenge was addressed by the GARPUR project 2017 (GARPUR, 2017).

\subsection{How will the future power system risk develop?}

New production with challenging properties, like solar and wind are put into the power system in an increasing rate. These alone will increase the risk in the system due to the characteristic intermittency property. It is expected more severe weather affecting the power system, resulting in higher risk. It is expected higher peak load as consequence of electrifying transportation and petroleum production, and this will increase risk in period of peak loads. New smart grid technology like disconnection of loads when needed, will reduce the risk in the system. The IoT (internet of things) technology may also increase the challenge of balancing the system due to rapid in and out connection of load driven by new sets of criteria not known by the system operators. This technology alone represents a threat to the system based on the possibility that third parties can hack into the equipment and connect/disconnect technology without anybody noticing, resulting possible outage of large areas.

Furthermore, battery storage is being introduced. This will most likely reduce the risk of outages in the system. In addition, artificial intelligence and machine learning is already on our door step. This can both help the system if done right, or increase the risk done wrong.

All these factors combined is a large order for the human mind to process in real-time. It is often seen that the risk driver is the combination of many seemingly unrelated factors and events, and not a single cause and effect scenario.

Example: A given power system has a unique dynamic characteristic and inherit risk property. The power system will typically be in a N-0 situation in periods with high peak load. Depending on the size of the load(s) affected by the reduced power delivery reliability, the total system risk is affected (see Figure 1). Above the red line the expected "not delivered energy" exceeds the acceptable level in regards of expected cost due to outage or the power companies' goals. Increase the system load for the same power system and the risk for outage will increase (see Figure 2). By operating the same

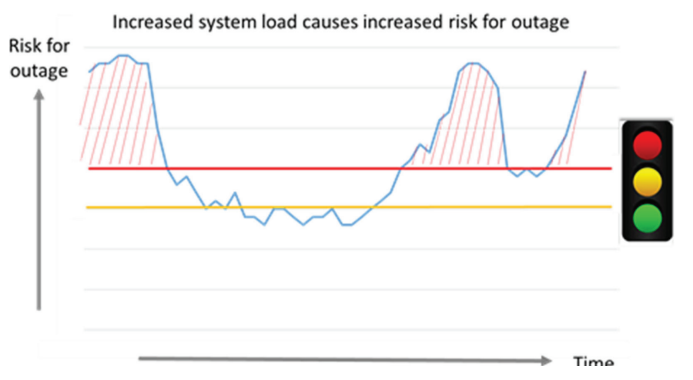

Figure 1. System risk for a given power system.

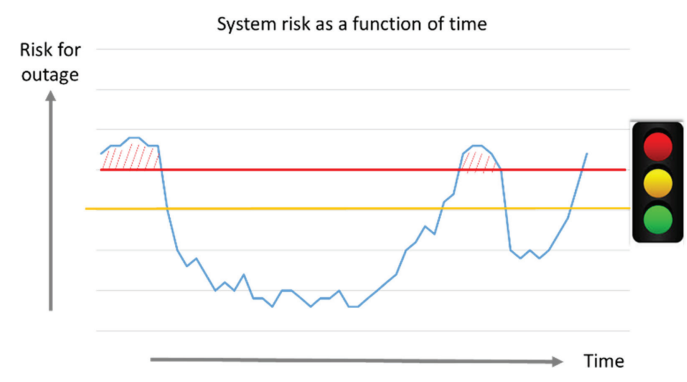

Figure 2. Increase only the load and the risk for outage increases. 


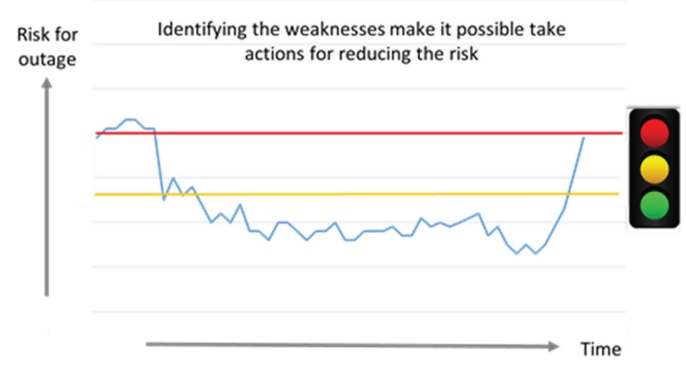

Figure 3. Operate the power system optimally, and the same increase in load can me managed in terms of risk for outage.

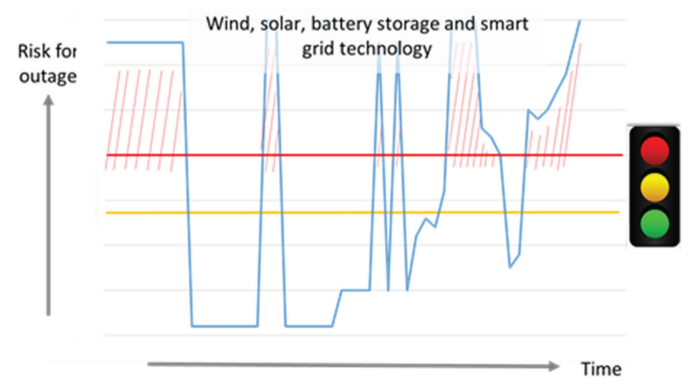

Figure 4. Prediction for future system risk development.

power system with the increased load more optimally, the total risk can be reduced to being within the acceptable risk level (see Figure 3).

With all the changes that are being introduced in the power system, our prediction is that the power system will experience system risk levels that goes from a very high level to the theoretical lowest level, and changing continuously by the minute (see Figure 4).

\section{PROBABILISTIC RISK ASSESSMENT}

\subsection{The starting point for a consistent risk management for the whole value chain}

To have a consistent risk management throughout to value chain, it is vital to be able to calculate the risk level of the current state of the power system. The current state will as it is in any power system analysis, be the base case for the next step analysis and evaluation. Furthermore, everything that is being done and planned, is being done for keeping the power system in operation. Therefore, the risk assessments done in the operation of a power system by operators or planning of operation, should be the foundation of risk evaluation to be commu- nicated in the power grid company as input for all future evaluations and analysis.

\subsection{Identifying the power system inherit risk properties}

To be able to identify the power system's inherent risk properties, the following objects and parameter has to be included in a mathematical representation of the power system:

- Production - Spinning reserve - Location

- Production type

- Power system configuration

- load flow

- load demand

- system dynamics

- Component reliability

- Maintenance interval and prioritizing

- Weather influence

- Energy storage possibilities and Smart grid technology

- System operators action and strategy

- Influence of other grid company's actions in their own power system

These factors have the characteristic of slow changing and fast changing properties. Since a power system is changing every minute of the year's 8760 hours, the total system risk graph will also vary by the minute. It is therefore, important to model the power system in great detail so that every change that occurs is reflected by the mathematical model. Furthermore, since historical data of the power system is stored (i.e. configuration, production, load flow, load level and failure rate for each component in the system) is available, validation of the mathematical model is possible to test against previous recorded risk levels.

\subsection{Calculation of the probabilistic risk level in near real time}

A short description of the calculation sequence in PROMAPS:

1. Calculate reliability of each grid segments. Each component in a grid segment can be described with multiple possible states, for instance Functioning, Intermediate fault or Lasting fault. PROMAPS use Markov models to represent each individual component in the grid segments:

$\dot{p}_{i}=A_{i} p_{i}$

where $A_{i}$ is a Markov model containing fault rates and repair rates.

2. It is possible to build reliability models of whole grid segments by simply combining all the 
Markov models of each individual component as Kronecker sums, as follows:

$$
A=A_{1} \oplus A_{2} \oplus \ldots \oplus A_{n}
$$

is combined into common states, thus reducing the number of states in the grid segment model to a few unique states.

3. Calculate the probability of each system states. A system reliability model is calculated by combining all grid segments models using Kronecher sums, as described in the last step.

4. Discard all system states with probability below some probability threshold. The probability threshold is dependent on how many states should remain in the set for further assessments.

5. Calculate maximum power transmission capacity for each state in set.

6. Calculate expected power shortage at each load point

7. Calculate expected power supply reliability, and mean time between loss of supply

8. Calculate various auxiliary variables including economic data.

The analysis can be performed for various load profiles. For online reliability assessments, parts of the calculation sequence are repeated whenever new online data is available.

Promaps risk assessment principles for online calculations has been presented in detail in PMAPS2012 (Svendsen, 2012). The concept has also been researched in the recently completed pan-European project GARPUR (GARPUR, 2017). Common for these methodologies for realtime risk assessments is that they consist of two main parts:

1. Calculate the probability of all sequences of events in the power grid

2. Calculate the consequence of these events.

Since there is a "infinite" number of possible events in a power grid, there also need to be some principle of discarding events with negligible risk. The simplest approach is to discard all contingency with probability below some probability threshold. The uncertainty of the risk assessments is related to the sum of risk of all events that has been discarded.

\section{AI AND MACHINE LEARNING IN OPERATION OF POWER SYSTEM}

\subsection{What data is needed}

There are numerous data available as input for AI and machine learning such as e.g.: current and historical: load, production, spinning reserve, sensor data (current, voltage, frequency) load flow, configuration of the power system, component data (type, characteristic, age), component health indexes, all previous outages and causes, weather type at the point of outage, dynamic data of the strength of the system form PMUs, protection schemes and other functionalities.

In addition, also near real time probabilistic risk calculation are available as input (Tollefsen, 2015). This represents big calculated data sets that gives a new insight in the inherit property of the power system.

Essential data for operation of the system: Voltage, frequency, production, spinning reserve and regulation possibilities.

\subsection{AI agents assigned to perform tasks in the power system}

Artificial Intelligence in general and supervised deep learning in particular tends to work well with large amounts of data (Schmidhuber, 2015). In supervised scenarios, the deep learning algorithms learn from known correct examples, and pick up trends and patterns that depict specific scenarios. In these cases, there is a trade-off between data quality and data size. The lower the quality of the data, the more data is needed extract the correct patterns. The most common example where this works is social media such as Facebook which contains enormous data of varying quality, enabling complex artificial intelligence algorithms.

The same basic concept is true for power systems, and it is therefore crucial that large amounts of data from power systems, including smart meters, is collected. The Norwegian power system manager Statnett has a particularly important role here. A concrete example of an application area artificial intelligence is expected to play a pivotal role is predicting electrical consumption peaks to avoid power outages (Goodwin, 2016). Over consumption may have serious consequences such as power outage. By predicting future peaks in the consumption, techniques such as load balancing could be carried out to avoid the problems. This clearly has to be carried out before the consumption peak happens, but knowing the consumption before occurrence is difficult. For this particular case, positive and negative examples should be collected, which in this case is examples of normal power flow, and over consumption. The artificial intelligence networks are trained with the data, and learns to understand which consumption trends lead to peak in the data. After the training phase, the network is put into practice and predicts future peaks which could either be used directly in an automated system to initiate load balancing, or as input to a decision support system. Other examples where artificial intelligence could play 
a similar role are operation of power system and production prediction.

The operation of a power system is based on a set of rules and constraints that the power system operator must operate the system within. The constraints are at set of limits that is related to the physical properties for the different power system components. This can e.g. be thermal limits for power lines/transformers or other components, the normal frequency deviation should be within $\pm 0,1 \mathrm{~Hz}$, voltage variations within $\pm 10 \%$ and the angle difference in three-phase current should be within maximum limits when reconnecting different part of the power system.

The rules and constraint connected to operation of a power system is notably different than the rules of board games such as chess. A power system is a stochastic system influenced by physical laws and human behavior such as consumption. The rules in a chess board and the behavior of the game are deterministic which means that future states are easily predictable, albeit many. However, there are similarities as well. The number waste amounts of states and complex behavior is identifiable in both complex board games and power systems. We can imagine that a machine learning also can be applied for operation of power systems based on the principle applied by Deep Mind with the new chees Alpha Zero program (Silver, 2017) and by the improved operation strategy obtained for Googles data center by use of Machine Learning Applications for Data Center Optimization (Gao, 2016).

\subsection{How can we evaluate the AI actions and gain trust?}

The artificial intelligence techniques vary from being statistically based on probabilistic induction, to knowledge based, and neuron based deep learning. For deep learning, which is undoubtedly, the most promising artificial intelligence technique in use, a confidence level is available as part of the supervised classification output. This confidence is very different from a probability, but can in any case be used as part of a trust schemes. If a deep learning network were to predict future problems, whenever it outputs an expected problem it can at the same time output how confident it is that is an actual problem. If this is part of a decision support system, the confidence can be used to inform a human decision maker in a decision support control room.

\section{HUMANS, DECISION SUPPORT SYSTEM AND AI}

\subsection{Decision support for system operation}

The ability to deal with the real-time fluctuations of the power system is not only a question of creating new technology and algorithms. Humans are still in the loop and the energy system is thus not only a technical system. It is a socio-technical system where the sense making, decisions and interventions of control room operators play an important part in the reliability of the system as a whole. This means that decision-support technology can play a key role in upholding the security of supply, but also that we need to take into account the human part of decision-making in control rooms. New decision-support systems will meet existing competence and experience, both at the individual and team level. In order to make sure that decision-support systems have the intended effects, the human perspective must be included in the development of the systems to ensure a good match between humans, technology and the organization of decision-making.

Advances in modelling and machine learning allow for information processing and problem solving that surpasses the capacity of an individual human decision-maker. Nevertheless, there is a need to find a balance between man and machine in the distribution of decision-making functions. Also, any period of technological transition will face challenges related to the competence of the existing workforce in the use of new technology, as well as a warranted level of trust into what new technology can and cannot do.

The importance of taking into account the relationship between human decision-making and algorithms can be illustrated by an example from New Scientist Volume 236, describing the domination of robots in the financial markets resulting in the human trader era is fading: "There are still a lot of unanswered questions surrounding the last bond market 'flash crash'. On 15 October 2014", "the US Treasury market crashed for about 10 minutes. Experts hypothesized that "activities of electronic trading algorithms" bore part of the blame, but reserved judgement for when they had more information. Three years later, no one is any wiser" (Adee, 2017).

This can also be the case for the future power system where smart grid technology, IoT and machine learning is put into operation. In order to avoid similar algorithm-induced "flash crashes" in the power system, it is vital to ensure the understanding of the system dynamics, the inherent risk properties and applying deep learning approaches as decision support.

\section{CONCLUSIONS}

The power system is rapidly changing towards the digital power system by using advanced ICT solutions, big data, smart grid, AI, machine learning and other advanced instruments. 
The digitalization of the power business in Norway, Europe and other parts of the world, arise numerous new possibilities but also challenges.

To gain trust in the machine learning technology being introduced to power system, and to avoid similar problems in the power system as the financial markets experienced with the 'flash crash' from 2014, new insight is needed. The need for understanding and tracking in near real time the power systems inherent system property in regards of power system dynamics and risk level, becomes evident.

\section{REFERENCES}

Adee S. 2017. The money machine. New Scientist, Volume 236, Issue 3147, 14 October 2017, Pages 22-23.

Aranya Chakrabortty, Alex Huang: 'Digital Grid: Transforming the Electric Power Grid into an Innovation Engine for the United States', North Carolina State University, Computing Catalyst Consortium 2017.

'Digitalization \& Energy', IEA, Sept 17, 2017.

Gao J. 2016. Machine Learning Applications for Data Center Optimization. Google 2016.

GARPUR 2017. Collaborative R\&D project co-funded by the European Commission (7th Framework Programme).

Gjengedal T.: *Power System Operation and Control, UiT-The Arctic University, 2017.

Goodwin M., A. Yazidi 2016. Journal: Integrated Computer-Aided Engineering, vol. 23, no. 2, pp. 101-113, 2016.

L'Heureux A., K Grolinger, H F. Elyamany, M A. M. Capretz 'Machine Learning With Big Data: Challenges and Approaches'. Machine Learning With Big Data: Challenges and Approaches' IEEE Access, April 2017.
NVE: 'New technologies and demand response'. NVE 2016.

Ould Amrouche S., D.Rekioua, T. Rekioua, S.Bacha: 'Overview of energy storage in renewable energy system'. International Journal of Hydrogen Energy, Volume 41, Issue 45, 7 December 2016, pages 20914-20927.

Rajendra Kumar Pandey, Mohit Misra 'Cyber Security threats-Smart grid infrastructure', Power Systems Conference (NPSC), 2016 National, India, IEEE Xplore: 20 February 2017.

Regulations on system responsibility in the power system (FOS) 2002, Ministry of Petroleum and Energy, Norway.

Reiten E., L. Sørgard, K. Bjella 2014. A better organized power grid, Ministry of Petroleum and Energy, Norway.

Schmidhuber J. 2014. Deep Learning in Neural Networks: An Overview. Journal reference: Neural Networks, Vol 61, pp 85-117, Jan 2015.

Silver D., T. Hubert, J. Schrittwieser, I. Antonoglou, M. Lai, A. Guez, M. Lanctot, L. Sifre, D. Kumaran, T. Graepel, T. Lillicrap, K. Simonyan, D. Hassabis 2017. Mastering Chess and Shogi by Self-Play with a General Reinforcement Learning Algorithm. Cite as: arXiv:1712.01815 [cs. AI].

"Smart Grids European Technology Platform" www. smartgrids.eu.smartgrids.eu. 2011. Retrieved 2011-10-11.

Statnett Grid Development Plan 2017 (in Norwegian). www.Statnett.no.

Svendsen A.B., J. Eman, T. Tollefsen, Y Aabø, T. Digernes, S. Løvlund, J. O. Gjerde 2012. Online reliability assessment of power system, PMAPS 2012.

Tollefsen T., A.B. Svendsen, R.F. Pedersen, P. Skeie, T.M. Lunde, J. Mælan 2015. Online Reliability Calculations of Power Systems with Forecasted and Real Time Weather Influence, Esrel 2015. 